Pediat. Res. 2: 22-28 (1968)

$\begin{array}{lll}\text { Aldosterone } & \text { hydrochlorothiazide } & \text { renin } \\ \text { angiotensin } & \text { kidney } & \text { vasopressin } \\ \text { diabetes insipidus, } & \text { potassium, } & \\ \text { nephrogenic } & \text { exchangeable } & \end{array}$

\title{
Etude du métabolisme de l'aldostérone dans un cas de diabète insipide néphrogénique congénital
}

\author{
C. Godard ${ }^{[27]}$, A. Mégevand, R. G. De Sousa et A.F. Muller \\ Clinique Universitaire de Pédiatrie et Laboratoire de Physiopathologie Clinique, Geneva, Switzerland
}

Extract

We have found repeatedly low or low-normal levels of aldosterone excretion in the urine of a boy presenting at birth with vasopressin-resistant diabetes insipidus. The only renal disorder was a lack of urinary concentrating ability.

A satisfactory gain of weight and height and a normal psychological development were obtained by use of a salt-free diet and small doses of hydrochlorothiazide administered during 18 consecutive months.

Aldosterone excretion ( $<1$ to $7.3 \mu \mathrm{g} / 24 \mathrm{~h}$ ) and secretion $(40 \mu \mathrm{g} / 24 \mathrm{~h})$ were surprisingly low, in view of sodium deprivation and thiazide treatment. Plasma renin activity was $53 \mathrm{ng} / \mathrm{l} / \mathrm{min}$, a value compatible with sodium restriction.

Angiotensin infusion over a period of 8 hours produced only a small increase of urinary excretion of aldosterone $(7 \mu \mathrm{g} / 12 \mathrm{~h})$. In a normal child of the same age, an 8-hour infusion of angiotensin raises urinary aldosterone excretion to $11.3 \mu \mathrm{g} / 12 \mathrm{~h}$ on a daily intake of $40 \mathrm{mEq} \mathrm{Na}$ and to $37 \mu \mathrm{g} / 12 \mathrm{~h}$ on an intake of $5 \mathrm{mEq} \mathrm{Na}$.

Our patient showed a slight increase of exchangeable sodium $(54.9 \mathrm{mEq} / \mathrm{kg}$ at 14 months and $53.1 \mathrm{mEq} / \mathrm{kg}$ at 3 years) and of bromide-space (27.9\% of total body weight), and a markedly decreased exchangeable potassium (23.9 and $24.5 \mathrm{mEq} / \mathrm{kg}$ ), without hypokaliemia.

\section{Speculation}

These results suggest that the existence of permanently increased total body stores of sodium in the presence of potassium depletion may decrease the production of aldosterone in the face of sodium restriction.

\section{Introduction}

Un des problèmes les plus troublants du diabète insipide néphrogénique (DIN) est celui que pose la relation entre l'insensibilité tubulaire à la vasopressine et la régulation du sodium. L'action thérapeutique favorable des salurétiques de la série des thiazides est ac- tuellement reconnue par tous. Son mécanisme pathogénique a fait l'objet de nombreuses discussions; la possibilité d'un antagonisme avec les minéralocorticoïdes au niveau du rein, évoqué par CRAwFord et al. [10], a été écartée par d'autres auteurs [12, 25, 26].

Par ailleurs, l'excrétion urinaire et la sécrétion d'aldostérone n'ont pas été étudiées très souvent dans le 
DIN. Friss-Hansen et al. [14] dans deux cas, Gautier [15] dans un cas, Brown et al. [5] dans un cas et l'un de nous (A.F.M.) dans plusieurs cas ont trouvé des aldostéronuries normales ou basses.

Nous avons eu l'occasion de déterminer de façon répétée l'excrétion urinaire d'aldostérone chez un enfant qui présentait dès sa naissance un diabète insipide résistant à la vasopressine: les valeurs obtenues étaient étonnamment basses, compte tenu, d'une part, d'une tendance à la déshydratation et, d'autre part, du régime pauvre en $\mathrm{NaCl}$. Nous en présentons ici les résultats ainsi qu'une étude sur l'effet de l'angiotensine et de l'ACTH. Une analyse isotopique de la composition électrolytique corporelle de notre patient a également pu être réalisée à deux moments différents (14 mois et 3 ans).

\section{Méthodes}

Les analyses chimiques, sanguines et urinaires, ont été faites selon les techniques courantes. Les osmolalités ont été mesurées avec l'osmomètre de Fiske.

L'excrétion et la sécrétion d'aldostérone ont été déterminées par la méthode de dérivation-dilution isotopique de Kliman et Peterson [18]. L'activité de la rénine plasmatique a été mesurée par le $\mathrm{Dr}^{\mathrm{R}} \mathrm{R}$. VeYrat avec la méthode biologique de Boucher et al. [4]; elle est exprimée en nanogrammes d'angiotensine libérée par litre de plasma par minuted'incubation ( $\mathrm{ng} / \mathrm{l} / \mathrm{min}$ ).

La détermination isotopique du sodium échangeable, du potassium échangeable et de l'espace-brome a été effectuée par le Laboratoire des Isotopes du Laboratoire Central de l'Hôpital Cantonal, Genève, selon un procédé décrit en détail par Busset et al. [6], appliquant l'injection simultanée de $\mathrm{Na}_{24}, \mathrm{~K}_{42}$ et $\mathrm{Br}_{82}$. La radioactivité injectée était inférieure à $100 \mu \mathrm{c}$.

La détermination des apports alimentaires de sodium a été faite par analyse chimique directe ou par calcul d'après des tables [11]. La corrélation entre les deux méthodes s'avéra bonne.

\section{Observation clinique}

R. G. ${ }^{\star}$ (Obs. Clin. Pediat.93078/65). Enfant unique de parents en bonne santé. Aucun cas de polyurie n'a été retrouvé dans la famille.

Toxicose gravidique de la mère. Accouchement à la 38 semaine le 28.8.1963, par césarienne pour souffrance fotale aiguë. Poids de naissance $=1800 \mathrm{~g}$. Asphyxie blanche néonatale, APGAR 1 à 2, réanimation pendant 10 minutes.

Les jours suivants l'enfant récupère bien; il est alimenté avec du lait acidifié et prend du poids. Des pous- sées fébriles intermittentes sont notées, ainsi qu'une hypertonie musculaire et une soif exagérée. La découverte d'une hypernatrémie à $171 \mathrm{mEq} / \mathrm{l}$ amène le transfert de la maternité à la Clinique de Pédiatrie à l'âge de 2 mois.

Le status d'entrée montre un nourrisson vif, pesant $3 \mathrm{~kg}$ mesurant $51 \mathrm{~cm}$ et présentant des signes discrets de déshydratation et une hypertonie musculaire diffuse avec hyperréflexie.

Examens. Chimisme sanguin: $\mathrm{Na}^{+}=162 \mathrm{mEq} / \mathrm{l}$, $\mathrm{K}^{+}=4,1 \mathrm{mEq} / \mathrm{l}, \mathrm{Cl}^{-}=122 \mathrm{mEq} / \mathrm{l}, \mathrm{HCO}_{3}^{-}=22$ $\mathrm{mEq} / \mathrm{l}$, azote uréique $=315 \mathrm{mg} / \mathrm{l}$. Osmolalité sanguine $=343 \mathrm{mOsm} / \mathrm{kg} \mathrm{H}{ }_{2} \mathrm{O}$. Calcémie $=10,2 \mathrm{~g} / \mathrm{l}$. Glycémie $=0,6 \mathrm{~g} / \mathrm{l}$. Protidémie $=6,2 \mathrm{~g} / \mathrm{l}$.

Urine: $\mathrm{pH} 5$ à 7 , albumine 0 . Sucre 0 . Sédiment sans particularités. Osmolalité urinaire $=70$ à $160 \mathrm{mOsm} /$ $\mathrm{kg}$.

Les jours suivants, l'hyperélectrolytémie persiste; le poids reste stationnaire et une polyurie modérée se manifeste (275 à $620 \mathrm{ml} / 24 \mathrm{~h}$ ). L'apport hydrique varie de 600 à $800 \mathrm{ml} / 24 \mathrm{~h}$ et celui de sodium de 7 à $8 \mathrm{mEq} / 24 \mathrm{~h}$. La natriurie est constamment basse, entre 2 et $8 \mathrm{mEq} / 24 \mathrm{~h}$. Les urines restent diluées et 2 unités de Pitressine ${ }^{\circledR}$ s.c. ne provoquent aucun changement de leur concentration osmolaire. Ce résultat, confirmé plus tard par d'autres épreuves à la vasopressine i.v. ou s.c., fait poser le diagnostic de diabète insipide néphrogénique congénital.

Un traitement d'hydrochlorothiazide est instauré à l'âge de 2 mois $1 / 2$ avec 25 puis 12,5 mg d'Esidrex ${ }^{\circledR}$ par jour. Il normalise rapidement et définitivement la natrémie et la chlorémie, alors que la natriurèse de $24 \mathrm{~h}$ s'élève temporairement. La diurèse n'est que peu modifiée. L'hypertonie musculaire disparait et la courbe de poids devient régulièrement ascendante. Les mois suivants, un régime riche en eau et pauvre en solutés est continué (apport de $\mathrm{Na}: 9 \mathrm{mEq} / 24 \mathrm{~h}$ en moyenne), de même que l'hydrochlorothiazide $(6,25$ à $12,5 \mathrm{mg} /$ jour $)$. On donne, en outre, un supplément journalier de potassium per os $(10 \mathrm{mEq})$.

A l'âge de 18 mois, l'enfant pèse $8 \mathrm{~kg}$ et mesure 75 $\mathrm{cm}$. Son développement psycho-moteur est absolument normal (test de BRunet-Lézine). Il n'existe plus d'hypertonie musculaire. La polydipsie est modérée: 1,5 à 2,51/jour. La formule sanguine et les électrolytes plasmatiques sont normaux.

Les différents examens effectués au cours de cette période n'ont pas révélé d'atteinte de la fonction rénale en dehors du défaut de concentration urinaire. La clearance de la créatinine endogène, déterminée plusieurs fois est normale ou légèrement abaissée: $128 \mathrm{ml} /$ $\min / 1,73 \mathrm{~m}^{2}$ à l'âge de 18 mois. L'aminoacidurie totale des urines est normale. Les natriurèses sont faibles, entre 1,5 et $4,5 \mathrm{mEq} / 24 \mathrm{~h}$. L'urographie i.v. est normale, à part un dédoublement pyélo-urétéral gauche, 
sans dilatation pyélo-calicielle. Cultures d'urines toujours négatives.

Depuis l'âge de 2 ans, l'enfant présente une anorexie importante et son poids est stationnaire aux environs de 10,5 kg. La croissance staturale est, en revanche, régulière $(80 \mathrm{~cm})$.

Le traitement consiste en régime pauvre en $\mathrm{NaCl}$, riche en eau, $25 \mathrm{mg}$ d'Hygroton ${ }^{\circledR}$ tous les 8 à 10 jours et un supplément quotidien de potassium.

A 3 ans (août 66), il mesure $88 \mathrm{~cm}$, mais ne pèse que $11 \mathrm{~kg}$. Il est toujours anorexique. Son Q. . est normal. On supprime le régime sans sel; l'appétit revient immédiatement. Le DIN est très bien équilibré avec $75 \mathrm{mg} /$ 24 h d'Edecrine ${ }^{\circledR}$.

A 2 ans 10 mois on effectue une ponction-biopsie rénale. La microscopie optique et électronique $\left(D^{r}\right.$ A. Chatelanat et $D^{r}$ A. Simon, Institut de Pathologie de l'Université de Genève) ne révèle aucune altération caractéristique d'une néphropathie. Le floculus des glomérules montre un épaississement irrégulier des membranes basales des anses capillaires et une tuméfaction modérée des cellules épithéliales. Pour autant qu'on puisse en juger, il n'y a pas d'hyperplasie des appareils juxtaglomérulaires (une description détaillée de cette histologie sera publiée ultérieurement).

Une épreuve à la Pitressine ${ }^{\circledR}$ chez la mère de l'enfant entraîne, avec 5 U s.c., une augmentation de l'osmolalité urinaire de 377 à $754 \mathrm{mOsm} / \mathrm{kg}$ en 3 heures.

\section{Etudes spéciales}

\section{Aldostérone}

Les résultats des dosages successifs de l'aldostérone urinaire sont rassemblés dans le tableau $\Upsilon$.

A l'âge de 2 mois et $2 \frac{1}{2} 2$ mois, l'enfant étant hyper- natrémique et déshydraté, l'aldostéronurie est de 3 et de moins de $1 \mu \mathrm{g} / 24 \mathrm{~h}$ respectivement.

Par la suite, ces taux restent à des valeurs normales pour l'âge [22], mais paradoxalement basses si l'on tient compte de la restriction alimentaire de $\mathrm{NaCl}$ dont la faible natriurie est la preuve chez un sujet en équilibre hydro-minéral. En effet, natrémie et kaliémie sont toujours normales pendant cette période.

A l'âge de 8 mois, sous régime pauvre en $\mathrm{NaCl}$, l'excrétion d'aldostérone est de $2,5 \mu \mathrm{g} / 24 \mathrm{~h}$; la sécrétion d'aldostérone est alors de $40 \mu \mathrm{g} / 24 \mathrm{~h}$ avec une natriurie de $1,5 \mathrm{mEq} / 24 \mathrm{~h}$. Rapporté à la surface corporelle, ce taux de sécrétion est de $133 \mu \mathrm{g} / 24 \mathrm{~h}$, soit une valeur un peut inférieure aux chiffres habituels chez un nourrisson recevant une alimentation normalement salée [16, $19,21]$.

A 18 mois, toujours sous régime désodé, l'aldostéronurie reste à $7 \mu \mathrm{g} / 24 \mathrm{~h}$. Au même moment, l'activité de la rénine plasmatique est de $53 \mathrm{ng} / \mathrm{l} / \mathrm{min}$.

\section{Stimulation de l'aldostérone par l'angiotensine II et l'ACTH}

Nous avons cherché à élever l'aldostéronurie par d'autres stimuli que la restriction sodique.

Les effets d'une perfusion i.v. d'angiotensine II sont représentés à la figure 1 . Les analyses urinaires ont été faites sur des périodes de 12 heures $(8 \mathrm{~h}-20 \mathrm{~h})$, à deux jours d'intervalle: une première fois sous perfusion de glucose à $5 \%$ (250 $\mathrm{ml}$ en 8 heures $)$, une seconde fois avec une perfusion identique contenant l'angiotensine (Hypertensine ${ }^{\circledR}$ ).

A gauche figurent les résultats de notre malade avec un régime pauvre en sodium ${ }^{1}: 200 \mathrm{mg}^{\text {d'angiotensine }}$

1 Pendant ces épreuves à l'ACTH et à l'angiotensine, notre malade suivait depuis plusieurs mois un régime sans sel et recevait, en moyenne 18,5 mg d'Esidrex/24 h.

Tableau I. Aldostéronuries de l'enfant R.C. (3-oxo-conjuguées, $\mathrm{pH}$ 1)

\begin{tabular}{|c|c|c|c|c|c|c|c|c|}
\hline $\begin{array}{l}\text { Age } \\
\text { mois }\end{array}$ & $\begin{array}{l}\text { Poids } \\
\mathrm{g}\end{array}$ & $\begin{array}{l}\text { Aldostérone } \\
\text { urine } \\
\mu \mathrm{g} / 24 \mathrm{~h}\end{array}$ & $\begin{array}{l}\text { Apport de } \\
\mathrm{Na} \\
\mathrm{mEq} / \text { jour }\end{array}$ & & $\begin{array}{l}\mathrm{K}+ \\
\text { ine } \\
/ 24 \mathrm{~h}\end{array}$ & & $\begin{array}{l}\mathrm{K}+ \\
\mathrm{rum} \\
\mathrm{Eq} / 1\end{array}$ & Remarques traitement \\
\hline 2 & 2900 & 3,0 & 7,0 & 2,0 & 6,0 & 166 & 3,6 & déshydraté \\
\hline $31 / 2$ & 3520 & 2,3 & 10 & 9,5 & 9,5 & 145 & 4,5 & $\begin{array}{l}\text { hydrochlorothiazide } \\
12,5 \mathrm{mg} / \mathrm{jour}\end{array}$ \\
\hline $41 / 2$ & 4400 & 4,8 & 10 & 4,5 & - & 143 & 4,1 & $\begin{array}{l}\text { hydrochlorothiazide } \\
12,5 \mathrm{mg} / \text { jour }\end{array}$ \\
\hline 8 & 5350 & 2,5 & 10 & 1,5 & 12 & 136 & 4,7 & (stop. thiazide depuis $48 \mathrm{~h}$ ) \\
\hline 18 & 8430 & 7,3 & 6,5 & 7,0 & 17 & - & - & $\begin{array}{l}\text { hydrochlorothiazide } \\
18,7 \mathrm{mg} / \text { jour }\end{array}$ \\
\hline 36 & 11000 & 5,2 & 10 & 15 & 30 & 142 & 5,1 & $\begin{array}{l}\text { chlorothiazide } \\
25 \mathrm{mg} 1 \times \text { par semaine }\end{array}$ \\
\hline
\end{tabular}




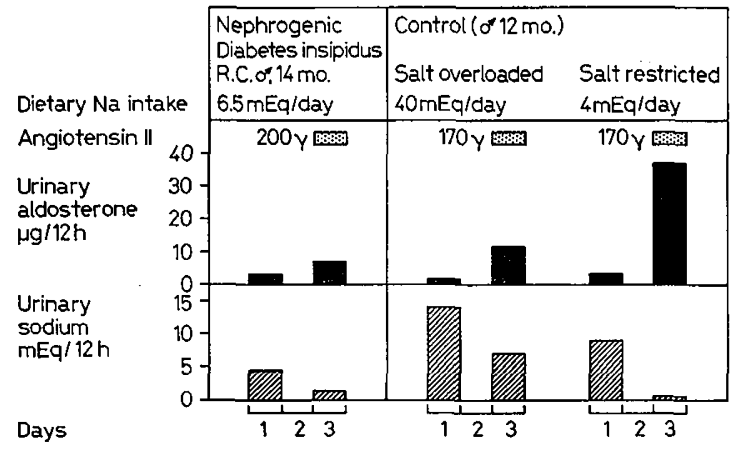

Fig. 1. Comparaison de l'effet d'une perfusion d'angiotensine sur l'excrétion urinaire d'aldostérone chez le patient et chez un sujet témoin. (Chez le témoin soumis au régime sans sel, la perfusion d'angiotensine [day 3] correspond au $4 \mathrm{e}$ jour de restriction sodée.)

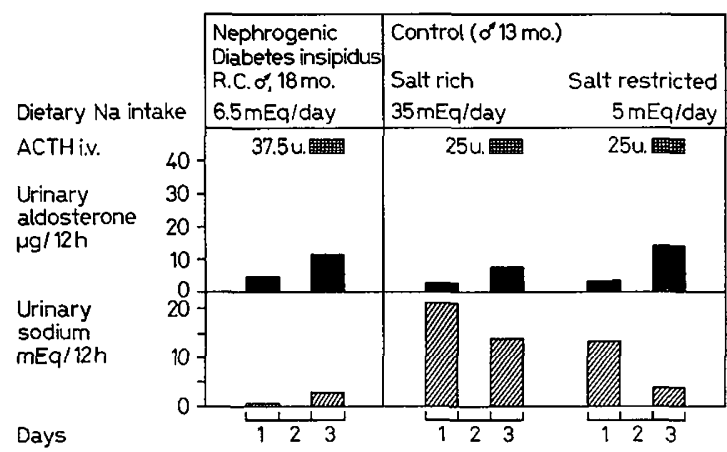

Fig. 2. Comparaison de l'effet d'une perfusion d'ACTH sur l'excrétion urinaire d'aldostérone chez le patient et chez un sujet témoin, dans les mêmes conditions que pour l'expérience de la figure 1 . i.v. élèvent l'aldostéronurie à $7 \mu \mathrm{g} / 12 \mathrm{~h}$; la natriurie s'abaisse à $1,5 \mathrm{mEq} / 12 \mathrm{~h}^{2}$.

Au centre est représentée la même épreuve chez un enfant normal du même âge, recevant une alimentation riche en sodium ( $40 \mathrm{mEq} / \mathrm{jour})$ : sous angiotensine, l'aldostéronurie s'élève à $11,3 \mu \mathrm{g} / 12 \mathrm{~h}$. A droite, la même épreuve sous restriction sodique chez le même témoin montre au quatrième jour une augmentation à $37 \mu \mathrm{g} / 12 \mathrm{~h}$.

Une stimulation par l'ACTH a également été réalisée chez notre patient selon un procédé identique (fig. 2). Sous perfusion i.v. de 37,5 UI d'ACTH (Gortrophin ${ }^{\circledR}$ ) dans $150 \mathrm{ml}$ de glucose à $5 \%$ pendant 8 heures, l'aldostéronurie passe à $11,5 \mu \mathrm{g} / 12 \mathrm{~h}$ et la natriurèse à $3 \mathrm{mEq} /$ $12 \mathrm{~h}$. Chez un enfant normal de 13 mois mis à une diète moyennement riche en sodium ( $35 \mathrm{mEq} / \mathrm{jour}$ ), 25 UI d'ACTH i.v. élèvent l'aldostéronurie à 7,5 $\mu \mathrm{g} /$ $12 \mathrm{~h}$; sous restriction sodique, l'ACTH élève l'aldostérone à $14 \mu \mathrm{g} / 12 \mathrm{~h}$ (quatrième jour).

\section{Sodium échangeable, potassium échangeable et espace- brome}

Les valeurs obtenues chez notre patient ainsi que chez trois enfants normaux de 9,12 et 12 mois, figurent dans le tableau II.

${ }^{2}$ Les effets de l'angiotensine sur la concentration des urines ont aussi été étudiés dans notre cas (perfusion i.v. continue d'Hypertensine ${ }^{\circledR}, 1 \mu \mathrm{g} / \mathrm{min}$ ). Comme cela a été observé chez l'adulte normal [20] et dans le DIN [6], le débit urinaire et la clearance de l'eau libre se sont abaissés, alors que l'osmolalité urinaire s'est élevée de 87 à $236 \mathrm{mOsm} / \mathrm{kg}$ en 2 heures.

La clearance de la créatinine endogène est restée inchangée.

Tableau II. Potassium échangeable $\left(\mathrm{K}_{\mathrm{e}}\right)$, sodium échangeable $\left(\mathrm{Na}_{\mathrm{e}}\right)$ et espace-brome

\begin{tabular}{|c|c|c|c|c|c|}
\hline & \multicolumn{2}{|c|}{$\begin{array}{l}\text { Diabète insipide } \\
\text { néphrogénique }\end{array}$} & \multicolumn{3}{|c|}{ Contrôles } \\
\hline & $\begin{array}{l}\text { R.G. } \\
o^{*} 14 \text { mois }\end{array}$ & 3 ans & I 12 mois & $\begin{array}{l}\text { II } \\
\text { o } 9 \text { mois }\end{array}$ & $\begin{array}{l}\text { III } \\
\text { of } 12 \text { mois }\end{array}$ \\
\hline Poids (kg) & 7,0 & 11,0 & 8,2 & 8,4 & 9,8 \\
\hline $\mathrm{K}_{\mathrm{e}}(\mathrm{mEq} / \mathrm{kg})$ & 23,9 & 24,5 & 36,3 & 33,2 & \\
\hline $\mathrm{Na}_{\mathrm{e}}(\mathrm{mEq} / \mathrm{kg})$ & 54,9 & 53,1 & 47,3 & 46,7 & 438 \\
\hline Espace-Brome (L) & 1,9 & & 2,1 & 2,1 & 2,6 \\
\hline$\%$ poids total & 27,9 & & 25,6 & 25,0 & 26,5 \\
\hline Eau total $(\mathrm{L})$ & & & 4,8 & 4,8 & \\
\hline$\%$ poids total & & & 58,5 & 57,1 & \\
\hline Formule d'F & & & & & \\
\hline Formule d'EDELMAN: $\overline{\mathrm{TBW}}$ & & & 142,2 & 139,8 & \\
\hline Natrémie (mEq/l) & 142 & 145 & 140,5 & 139,5 & \\
\hline
\end{tabular}

Dans le DIN, l'eau totale (méthode de dilution du tritium) n'a pas été mesurée, pour ne pas gêner des analyses stéroïdiennes ultérieures utilisant le marcage avec $\mathrm{H} 3$. 
Le potassium échangeable $\left(\mathrm{K}_{\mathrm{e}}\right)$ de $23,9 \mathrm{mEq} / \mathrm{kg}$ est nettement abaissé. La valeur de $54,9 \mathrm{mEq} / \mathrm{kg}$ pour le sodium échangeable $\left(\mathrm{Na}_{\mathrm{e}}\right)$ est un peu trop élevée par rapport à celle de nos enfants témoins. L'espace-brome paraît également un peu augmenté. Ces différences sont peut-être à mettre, en partie, sur le compte de l'état de nutrition déficitaire de notre malade, car les chiffres sont exprimés par rapport au poids corporel total et non à la «masse maigre» [8]; le pli cutané abdominal était mince ${ }^{3}$.

\section{Discussion}

Le diagnostic de diabète néphrogénique congénital, non associé à une néphropathie plus complexe, est assuré. (La découverte d'une duplication pyélo-urétérale est fortuite et sans relation avec le DIN.) L'anamnèse familiale détaillée fait penser qu'il s'agit d'une forme sporadique de DIN.

Les excrétions urinaires d'aldostérone sont normales pour l'âge [22]. Le taux de sécrétion paraît également dans les limites normales. Mais ces résultats sont surprenants, car avant tout traitement l'enfant était déshydraté ( $\mathrm{Na} 162$ à $171 \mathrm{mEq} / \mathrm{l}$ ) et par la suite, il recevait

${ }^{3}$ Pour ces analyses isotopiques, le traitement diurétique (12,5 mg Esidrex ${ }^{\circledR}$ par jour) avait été interrompu depuis $24 \mathrm{~h}$. un régime pauvre en sodium associé à un traitement continu d'hydrochlorothiazide. L'une et l'autre de ces deux situations devraient élever l'excrétion et la sécrétion d'aldostérone [2].

Chez d'autres enfants atteints de DIN (tableau III), des aldostéronuries basses ont également été trouvées malgré la restriction saline et les salurétiques [5, 14, 15]. Les valeurs du sodium urinaire témoignent, chez les sujets en équilibre hydro-minéral, de la restriction du sel. Dans de telles conditions, les aldostéronuries sont toujours supérieures aux taux trouvés chez les malades atteints de DIN. On a, peut-être, dans cet hypoaldostéronisme, l'explication de l'inefficacité des spirolactones comme agents diurètiques dans le DIN [12, 25, 26]. Dans le premier cas de Frus-Hansen, l'aldostérone s'élève cependant à $23 \mu \mathrm{g} / 24 \mathrm{~h}$ après un traitement prolongé de thiazide + spirolactone. Dans le cas de BROWN, elle passe de 8 à $57 \mu \mathrm{g} / 24 \mathrm{~h}$ après 4 jours d'acide éthacrynique; simultanément, le volume plasmatique s'abaisse de 1373 à $1139 \mathrm{ml}$, ce qui démontre indirectement l'effet de ce salurétique sur le capital sodique du malade.

La non-réponse, dans la majorité des cas de DIN, de l'aldostérone à la mise au régime sans sel et aux salurétiques peut être due au fait que ceux-ci n'entraînent pas un degré de déplétion sodique suffisant. L'activité de la rénine plasmatique de notre patient $(53 \mathrm{ng} / \mathrm{l} / \mathrm{min}$ ) semble pourtant compatible avec une telle déplétion. Chez l'adulte normal recevant une alimentation

Tableau III. Aldostéronuries dans le diabète insipide néphrogénique

\begin{tabular}{|c|c|c|c|c|c|}
\hline Auteur & Cas, âge & $\begin{array}{l}\text { Aldostérone } \\
\text { urine }(\mathrm{pH} 1) \\
\mu \mathrm{g} / 24 \mathrm{~h}\end{array}$ & Apport de $\mathrm{Na}$ & $\begin{array}{l}\mathrm{Na}+ \\
\text { urine } \\
\mathrm{mEq} / 24 \mathrm{~h}\end{array}$ & Traitement \\
\hline FrISS-HANSEN et al. [14] & $\begin{array}{l}\text { o } 3 \text { ans } \\
\text { t } 8 \text { mois }\end{array}$ & $\begin{array}{c}1,9 \\
2,4 \\
23 \\
1,0\end{array}$ & $\begin{array}{l}\text { faible } \\
\text { faible } \\
\text { normal } \\
\text { normal }\end{array}$ & $\begin{array}{r}5 \\
30 \\
25\end{array}$ & $\begin{array}{l}\text { thiazide } \\
\text { thiazide + spirolactone } \\
\text { - }\end{array}$ \\
\hline Gautier [15] & of 5 ans $1 / 2$ & $\begin{array}{l}6,3 \\
6,5 \\
7,4 \\
\end{array}$ & $\begin{array}{l}\text { faible } \\
\text { faible } \\
\text { normal }\end{array}$ & $\begin{array}{r}18 \\
14 \\
2 \\
\end{array}$ & thiazide \\
\hline BRown et al. [5] & o 9 ans & $\begin{array}{c}8,0 \\
57\end{array}$ & $\begin{array}{l}60 \mathrm{mEq} / \text { jour } \\
60 \mathrm{mEq} / \text { jour }\end{array}$ & $\begin{array}{l}30 \\
40\end{array}$ & $\begin{array}{l}\text { - } \\
\text { acide éthacrynique } \\
\left(4 \mathrm{e}^{\mathrm{e}} \text { jour }\right)\end{array}$ \\
\hline Cas personnels & $\begin{array}{l}\text { o } 2 \text { ans } \\
\text { o } 14 \text { mois }{ }^{1} \\
\text { o } 5 \text { ans } \\
\text { o } 5 \text { ans } \\
\text { o } 3 \text { ans }{ }^{2}\end{array}$ & $\begin{array}{r}2,8 \\
2,2 \\
6,6 \\
2,2 \\
8,8 \\
14\end{array}$ & $\begin{array}{l}\text { faible } \\
\text { faible } \\
\text { faible } \\
\text { faible } \\
\text { faible } \\
\text { faible }\end{array}$ & $\begin{array}{r}15 \\
3 \\
5 \\
30 \\
8\end{array}$ & $\begin{array}{l}- \\
- \\
\text { thiazide } \\
\text { thiazide }\end{array}$ \\
\hline
\end{tabular}

${ }^{1}$ Sécretion d'aldostérone $=71 \mu \mathrm{g} / 24 \mathrm{~h} \quad{ }^{2}$ Cas publié par Weber et Gautier [26] 
moyennement salée, l'activité de la rénine plasmatique est de $9,0 \pm 7,6 \mathrm{ng} / \mathrm{l} / \mathrm{min}$ [24]. Cette activité augmentée de la rénine plasmatique dans le DIN indiquerait que le système rénine-angiotensine réagit lui-même au stimulus de la déplétion sodique (les valeurs non élevées d'aldostérone urinaire sont d'autant plus étonnantes). Il faut cependant interpréter ces chiffres avec prudence, car on a peu d'expérience des taux d'activité de la rénine chez des nourrissons et petits enfants sains recevant une alimentation moyennement salée; il semblerait que les valeurs soient plus fluctuantes à cet âge que chez l'adulte.

Parmi les différents stimuli directs de la sécrétion d'aldostérone, l'angiotensine II est l'un des plus puissants $[3,20]$. Chez notre patient, l'angiotensine i.v. a provoqué une augmentation certaine de l'aldostéronurie (fig. 1), mais inférieure à celle que l'on observe chez un enfant normal légèrement surchargé en $\mathrm{NaCl}$. Ce qui est surtout frappant, c'est la comparaison avec un enfant normal au régime sans sel strict. Quant à la réponse de l'aldostérone à l'ACTH i.v., elle a été plus nette et les différences avec le normal plus petites (fig.2).

Pourquoi n'y a-t-il pas d'hyperaldostéronisme en cas de restriction saline dans le DIN? Il est difficile de l'expliquer, mais l'étude de la composition électrolytique corporelle permet d'apporter quelques éclaircissements à cette question.

a) Il a été démontré que la déplétion potassique empêche la réponse de l'aldostérone au stimulus de la déplétion sodique $[1,20]$. Ce facteur est à prendre en considération dans le DIN, car, dans notre cas, le $\mathbf{K}_{e}$ est nettement abaissé. L'absence d'hypokaliémie n'exclut pas un rôle inhibiteur de la déplétion potassique [7]. Dans quelle mesure une éventuelle carence potassique pourrait jouer un rôle chez le sujet non traité est impossible à dire.

b) Une augmentation du sodium total de l'organisme et de l'espace extra-cellulaire (suggérée par le résultat du $\mathrm{Na}_{\mathrm{e}}$ et de l'espace-brome) pourrait également exercer un effet inhibiteur sur la production d'aldostérone. Dans notre cas, le $\mathrm{Na}_{\mathrm{c}}$ de $54,9 \mathrm{mEq} / \mathrm{kg}$ représente une valeur élevée, si on la compare avec celles de nos enfants témoins (tableau II).

Forbes et Perley [13] et Corsa et al. [9] ont trouvé chez des enfants normaux de cet âge, des chiffres de $\mathrm{Na}_{\mathrm{e}}$ nettement plus élevés que les nôtres, mais une comparaison avec les données de la littérature s'avère difficile, les méthodes d'analyses n'étant pas absolument identiques. La parfaite concordance, chez les sujets-contrôles I et II entre la formule d'EDELMAN et la natrémie obtenue par dosage, ainsi que la confirmation apportée par les déterminations faites chez notre patient R.C. à 3 ans, nous autorise à interpréter le $\mathrm{Na}_{\mathrm{e}}$ de ce DIN comme étant élevé.

S'il en est ainsi, ce résultat suggère une augmentation du capital sodique de l'organisme. La cause n'en est pas claire. Il est possible (mais non prouvé) que l'excès d'hormone antidiurétique (ADH) circulante existant dans le DIN [17] intervienne en favorisant une rétention primaire de sodium. Des expériences sur la muqueuse vésicale de la grenouille ont mis en évidence une action propre de l'ADH sur le transport cellulaire du sodium, indépendamment de son influence sur la perméabilité à l'eau [23]. Chez l'homme, une telle action n'a pas été démontrée, mais seulement suggérée par analogie. Par ailleurs, si un effet propre de l'ADH sur le sodium existe réellement, on ignore s'il est conservé ou non dans le DIN.

Etant donné que les deux analyses isotopiques ont été réalisées après un traitement long et continu aux diurétiques, leur interprétation doit se faire avec prudence. La discordance de nos valeurs avec celles de Fris-Hansen est peut-être méthodologique; elle ne nous paraît pas capitale vu nos propres contrôles.

En conclusion, un $\mathrm{Na}_{\mathrm{e}}$ augmenté et un $\mathrm{K}_{\mathrm{e}}$ diminué nous paraissent constituer des modifications biologiques capables, l'une et l'autre, de rendre la couche glomérulaire de la surrénale moins sensible («less responsive») aux stimuli habituels tels que la restriction sodique ou la perfusion d'angiotensine. Cette hypothèse devra être ultérieurement vérifiée.

\section{Conclusion et résumé}

Des aldostéronuries basses ont été trouvées à plusieurs reprises chez un garçon qui présentait dès la naissance un diabète insipide néphrogénique pur. Une croissance staturo-pondérale satisfaisante et un développement psycho-moteur normal ont été obtenus grâce à un régime pauvre en $\mathrm{NaCl}$ et un traitement d'hydrochlorothiazide poursuivis pendant 18 mois.

Les excrétions urinaires et la sécrétion d'aldostérone étaient étonnamment basses, compte tenu de la restriction saline et du traitement salurétique. L'angiotensine i.v. n'a entraîné qu'une faible augmentation de l'aldostéronurie. Le taux d'activité de la rénine plasmatique était, en revanche, compatible avec un régime sans sel.

La découverte d'un sodium échangeable un peu augmenté et d'un potassium échangeable nettement diminué laisse penser que ces deux facteurs peuvent, dans ce cas de diabète insipide néphrogénique, contribuer à la faible réponse de l'aldostérone lors de la mise au régime sans sel.

\section{Références et notes}

1. Bartter, F. G.; Barbour, B.H.; Garr, A.A. and Delea, C.S.: On the role of potassium and of the central nervous system in the regulation of aldoste- 
rone secretion; in: Aldosterone, éd. Baulrev, E. E. et Robel, P. (Blackwell, Oxford 1964).

2. Bartter, F. G.; Mills, I.H.; Biglieri, E. G. and Delea, C.: Studies on the control and physiologic action of aldosterone. Recent Progr. Hormone Res. 15: 311-334 (1959).

3. Biron, P.; Korw, E.; Nowaczynski, W.; BroullLET, J. and Genest, J.: The effects of intravenous infusions of valine -5 angiotensin II and other pressor agents on urinary electrolytes and corticosteroids, including aldosterone. J. clin. Invest. 40: 338-347 (1961).

4. Boucher, R.; Veyrat, R.; de Ghamplain, J. and Genest, J.: New procedures for measurements of human plasma angiotensin and renin activity levels. Canad.med. Ass. J. 90: 194-201 (1964).

5. Brown, D.M.; Reynold, J.W.; Mrchael, A.F. and Ulstrom, R.A.: The use and mode of action of ethacrynic acid in nephrogenic diabetes insipidus. Pediatrics 37: 447-455 (1966).

6. Busset, R.; De Sousa, R. C. et MAch, R.S. : Etude de la composition corporelle à l'aide de radioisotopes chez l'homme normal et au cours de différents états pathologiques. I. Méthode. Sem.Hôp., Paris 43: 1312-1321 (1967).

7. Cannon, P.J.; Ames, R.P. and Laragh, J.H.: Relation between potassium balance and aldosterone secretion in normal subjects and in patients with hypertension or renal tubular disease. J. clin. Invest. 45: 865-879 (1966).

8. CheEk, D.B.: Extracellular volume: its structure and measurement and the influence of age and disease. J.Pediat. 58: 103-122 (1961).

9. Gorsa, L.; Gribetz, D.; Gook, G.D. and TalBOт, N. B. : Total body exchangeable water, sodium and potassium in 'hospital normal' infants and children. Pediatrics 17: 184-191 (1956).

10. Crawford, J. D.; Kennedy, G. C. and Hill, L. E.: Clinical results of treatment of diabetes insipidus with drugs of the chlorothiazide series. New Engl. J.Med. 262: 737-747 (1960).

11. Diem, K.: Composition chimique des aliments; in: Tables scientifiques, pp.516-528 (Geigy, Bâle 1963).

12. EARLEY, L.E. and OrLoff, J.: The mechanism of antidiuresis associated with the administration of hydrochlorothiazide to patients with vasopressinresistant diabetes insipidus. J.clin. Invest. 41: 1988-1997 (1962).

13. Forbes, G. B. and Perley, A.: Estimation of total body sodium by isotopic dilution. II. Studies on infants and children. J.clin. Invest. 30: 566-574 (1951).

14. Fris-Hansen, B.; Skadhauge, E. and ZetterSTRÖM, R.: Fluid and electrolyte metabolism in nephrogenic diabetes insipidus. Two cases. Acta Paediat. (Uppsala) Suppl. 146: 57-67 (1963).

15. GAutrer, E.: Neonatal hyperosmolality, an instance of unresponsiveness to antidiuretic hormone. Nutritia Symposium. The adaptation of the newborn infant to extra-uterine life, pp.83-94 (Stenfert Kroese, Leiden 1964).

16. Godard, G.; Riondel, A.; Veyrat, R.; MégeVAND, A. and MULLER, A.F.: Plasma renin activity and aldosterone secretion rate in congenital adrenal hyperplasia. Pediatrics (in press).

17. Holliday, M.A.; Burstin, C. and Harrah, J.: Evidence that the antidiuretic substance in the plasma of children with nephrogenic diabetes insipidus is antidiuretic hormone. Pediatrics 32: 384388 (1963).

18. Kliman, B. and Peterson, R.E.: Double isotope derivative assay of aldosterone in biological extracts. J. Biol. Ghem. 235: 1639-1648 (1960).

19. Weldon, V.V.; Kowarski, A.; Migeon, G.J. and Ozolins, B.: Aldosterone secretion: Infancy to adulthood. Pediatrics 39: 713-723 (1967).

20. Laragh, J.H.; Cannon, P.J. ; Ames, R.P.; Sicinski, A. M. and BorkowsKY, J.: Aldosteronism in man: mechanisms controlling secretion of the hormone; role of angiotensin; in: Angiotensin and the kidney (ed. Metcoff, J.), pp. 108-128) (Little, Brown, Boston 1963).

21. Loras, B.; Do, F.; Forest, M.; Gautenet, B.; Frederich, F. and Bertrand, J.: Contribution to the study of the exchangeable sodium pool and of aldosterone secretion rates. Variations with age and during sodium deprivation. Communication to the European Glub for Pediatric Research (Athens 1966).

22. New, M.I.; Miller, B. and Peterson, R.E.: Aldosterone excretion in normal children and in children with adrenal hyperplasia. J.clin. Invest. 45: 412-428 (1966).

23. ORLOFF, J. and HANDLER, J. S.: The cellular mode of action of antidiuretic hormone. Amer.J.Med. 36: 687-697 (1964).

24. Veyrat, R.; de Champlain, J.; Boucher, R.; Korw, E. et Genest, J. : Le rôle du système rénineangiotensine dans la régulation du métabolisme du sodium chez l'homme. Schweiz.med.Wschr. 94: 914-922 (1964).

25. Weber, J.W. und Gautier, E. : Pitressinresistenter Diabetes insipidus: Therapie mit Salidiuretica. Helv. Paediat. Acta 16: 565-585 (1961).

26. Wolfe, A.J. and Werl, W.B.: Mechanism of the antidiuretic action of thiazide derivatives in diabetes insipidus. J. Pediat. 63: 764-766 (1963).

27. Godard, C., M.D., Clinique Universitaire de Pédiatrie, $30 \mathrm{Bd}$. de la Cluse, 1211 Genève 4 (Suisse). 